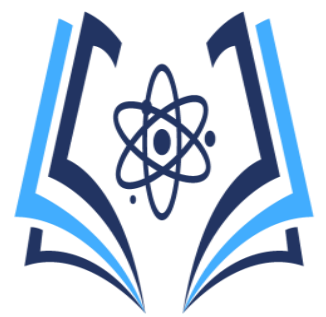

\title{
La homofobia y su influencia negativa en la salud mental
}

\section{Homophobia and its negative influence on mental health La homofobia y su influencia negativa}

\author{
Anny Salome Adrián Puya ${ }^{(1)}$ \\ Reynier García Rodríguez ${ }^{(2)}$ \\ María José Álvarez Moreira ${ }^{(3)}$ \\ Ana Paula García Intriago ${ }^{(4)}$ \\ ${ }^{1}$ Instituto Superior Universitario Portoviejo, Manabí, Ecuador. ORCID: https://orcid.org/0000-0001-5165-7991 \\ Email: annyadrian05@gmail.com \\ ${ }^{2}$ Instituto Superior Universitario Portoviejo, Manabí, Ecuador https://orcid.org/0000-0002-4160-5749 Email: \\ reygarod79@gmail.com \\ ${ }^{3}$ Instituto Superior Universitario Portoviejo, Manabí, Ecuador. ORCID: https://orcid.org/0000-0003-3181-3154 \\ Email: alvarez_0402@hotmail.com \\ ${ }^{4}$ Instituto Superior Universitario Portoviejo, Manabí, Ecuador. ORCID: https://orcid.org/0000-0002-1287-3218 \\ Email: anapaulagain@ hotmail.com
}

Contacto: annyadrian05@gmail.com

Recibido: 25-07-2021

Aprobado: 10-08-2021

\section{Resumen}

La homofobia es y ha sido una de las problemáticas más comunes alrededor del mundo. Por esta razón el objetivo del artículo es concientizar a la sociedad en torno a la lucha contra la homofobia promoviendo el respeto hacia todas las personas sin importar su género o su orientación sexual.

La homofobia se ve representada a través de actitudes y comportamientos discriminatorios en contra de la comunidad LGBTI, estas conductas tienen un efecto directo en esta comunidad ya que la mayoría de estos son rechazados y marginados, violentando sus derechos.

Se realizó un estudio de carácter descriptivo y de corte longitudinal, en el que se aplicó una encuesta de
10 preguntas a una muestra de 50 personas con el fin de comprender el conocimiento de la sociedad relacionado al tema de la homosexualidad y homofobia. Con los resultados se evidencio que a pesar que la mayoría tiene conocimiento sobre este tema no se absuelve a las personas de sufrir bullying por tener una orientación sexual diferente.

Las personas que han sufrido de ataques homofóbicos tienen problemas al desarrollarse socialmente, sentimentalmente y sexualmente, también tienden a tener adicciones a sustancias ilegales, a aceptar maltratos, burlas y ofensas debido a su baja autoestima, muchas han terminado en el suicidio debido a la discriminación. Por esta razón se debe fomentar el respeto y la tolerancia en todos los espacios, desde el hogar, planteles educativos $\mathrm{y}$ 
laborales promoviendo los derechos de todas las personas sin distinción.

Palabras clave: Homofobia, homosexualidad, salud mental, sexualidad, genero, respeto.

\begin{abstract}
Homophobia is and has been one of the most common problems around the world. For this reason, the objective of the article is to raise awareness in society about the fight against homophobia by promoting respect for all people regardless of their gender or sexual orientation.
\end{abstract}

Homophobia is represented through discriminatory attitudes and behaviours against the LGBTI community, these behaviours have a direct effect on this community as most of them are rejected and marginalized, violating their rights.

A longitudinal, descriptive study was conducted in which a 10-question survey was applied to a sample of 50 people in order to understand society's knowledge of the issue of homosexuality and homophobia. With the results it became evident that despite the fact that most people have knowledge about this subject, people are not absolved from suffering from bullying because they have a different sexual orientation.

People who have suffered from homophobic attacks have problems developing socially, emotionally and sexually, they also tend to have addictions to illegal substances, to accept mistreatment, ridicule and offense due to their low self-esteem, many have ended up committing suicide due to discrimination. For this reason, respect and tolerance should be encouraged in all spaces, from the home to educational and workplaces, promoting the rights of all people without distinction.

Keywords: Homophobia, homosexuality, mental health, sexuality, gender, respect.

\section{Introducción}

Durante mucho tiempo se creyó que la homosexualidad era una enfermedad mental, pero la
Vol. 5, Nro. 2, Publicado: 2021-12-31

OMS la descalifica como un trastorno mental. (OMS, 2017)

La homofobia es la representación actitudes y comportamientos que expresan incomodidad y rechazo que sienten las personas en contra de la comunidad LGBT, esto también se manifiesta a través de conductas discriminatorias, estas conductas van desde lo verbal, lo físico y en algunos casos puede haber homicidio hacia esta comunidad.

La homofobia tiene un efecto directo sobre esta población es decir que la mayoría son rechazados, marginados, no tienen acceso a empleos y muchas veces se les niega llevar una vida común violentando sus derechos. (Activismo Cultural y Derechos Humanos, 2017)

Se han insinuado varios orígenes de la homofobia: ya sean por cuestiones religiosas, miedo interiorizado a ser homosexual, celos cohibidos o la falta de valores; sin embargo, falta mucho por estudiar de este fenómeno. Todos los gestos homofóbicos son aprendidos o los adoptamos de otras personas. (Duarte, 2015)

En los gestos homofóbicos relacionados a la religión, se puntualiza la homofobia como una actividad antinatural, es decir promueve la heterosexualidad como lo normal, debido a que se considera que las personas que mantienen relaciones con otras de su mismo sexo son víctimas del pecado. Muchos líderes de las iglesias se refieren a las personas de esta comunidad con términos de odio y aversión, promoviendo el aborrecimiento por parte de la sociedad religiosa. (Scielo, 2019)

Las personas que han sufrido de ataques homofóbicos tienen dificultades para desarrollarse social, emocional y sexualmente, presentan adicciones a sustancias ilegales, también aceptan el maltrato, burlas y agresiones de personas cercanas ya que su nivel de autoestima es bajo.

La mayoría de estas personas tienen sentimientos de temor, ansiedad, preocupación ya que sufren de 
Periodo. Julio - Diciembre 2021

Vol. 5, Nro. 2, Publicado: 2021-12-31

burlas y discriminación en sus espacios laborales y familiares. Este modelo es una amenaza potencial para la salud mental y también la vida de la persona por tener pensamientos suicidas.

El 17 de mayo de 1900 se declaró oficialmente el Día internacional contra la homofobia, transfobia y bifobia, abona a la construcción de una sociedad que acepta la diversidad y reconoce los derechos humanos de todas las personas, independientemente de su orientación sexual e identidad de género

A nivel mundial existen 124 Estados donde no existen leyes que penalicen las relaciones sexuales consensuales entre personas adultas del mismo sexo. Existen 72 Estados que criminalizan las relaciones sexuales entre personas adultas del mismo sexo. En 45 de estos Estados (24 en África, 13 en Asia, 6 en América y 2 en Oceanía) estas normas criminalizan tanto a hombres como a mujeres. Aunque en 6 países se castiga la homosexualidad con pena de muerte, estos países son: arabia saudí, Irán, Yemen, Sudan, Nigeria y una parte de Somalia. (Samaniego \& Bermúdez, 2015)

En este sentido las leyes que protege a las personas lesbianas gais y bisexuales de la discriminación y la violencia se ha ampliado en los últimos años, aunque a un ritmo más lento de lo que se esperaba.

En Ecuador desde el año 1997 la homosexualidad no está penalizada por la ley, además desde el año 2019 existe la posibilidad del matrimonio igualitario, es importante destacar que la ley reconoce el principio de igualdad y no discriminación ya sean por motivos de orientación sexual o identidad de género, además la constitución ampara a todas las personas sin discriminación. A pesar de que en el ámbito legal no se discrimina a las personas, el maltrato a la comunidad LGBT es constante en pequeños grupos de la sociedad. (Otero, 2016)

En la provincia de Manabí existe demasiada discriminación, estas personas reciben piropos

Tabla 1. Conocimiento del término homofobia malintencionados, insultos, acoso, bullying. Para muchas personas el tono de voz, la forma de vestir, caminar, la manera de expresarse puede significar que dichas personas pertenecen a la comunidad LGBT. Entre los más vulnerados al maltrato físico, verbal, sexual, de esta comunidad son las personas transgéneros debido a su identidad de género y no específicamente por su orientación sexual.

La erradicación de la homofobia en la sociedad es una necesidad para poder desarrollar un círculo igualitario, centrándonos en promover valores desde el hogar, en escuelas y en los espacios laborales.

El objetivo del presente artículo es: Orientar a las personas acerca de la homofobia que existe en la sociedad.

\section{Materiales y métodos}

La población fue de 50 personas escogidas al azar, el estudio es de tipo descriptivo, la técnica utilizada fue una encuesta de 10 preguntas. Es un estudio de corte longitudinal en el periodo comprendido desde julio de 2020, en el cantón Portoviejo, de la provincia de Manabí, Ecuador.

En el estudio se encuestó a personas desde los 18 años, con el fin de indagar acerca de su conocimiento relacionado a la homofobia y las orientaciones sexuales, se delimitaron variables como la edad, el sexo, la situación económica y la etnia.

Se empleó una encuesta hecha con Formularios de Google, realizando un total de 10 preguntas, se utilizó WhatsApp y Messenger para difundir la encuesta y explicar el motivo de la misma.

Se procedió al diseño de una base de datos para poder realizar el análisis estadístico, se utilizó el programa Microsoft Office Excel. Se estimaron los porcentajes como medida de resumen de la estadística descriptiva y se estimaron las frecuencias relativas.

\section{Resultados}

La encuesta mostro resultados importantes para continuar la investigación, evidenciando lo siguiente: 


\begin{tabular}{|l|c|c|}
\hline Aspectos & Usuarios & $\%$ \\
\hline Sí & 37 & 90,2 \\
\hline No & 4 & 9,8 \\
\hline Total & 41 & 100 \\
\hline
\end{tabular}

La encuesta aplicada a la muestra seleccionada, indica que relacionado con el termino homofobia, un total de 37 personas que representaron el 90,2\%, revelan que si lo conocen. En tanto, 4 personas que representaron el 9,8\%, plantean que no lo conocen. El desconocer el termino homofobia, no exime de bullying a quienes tienen una orientación sexual diferente, esto induce a que debe ser investigado.

Tabla 2. Considerar si las personas homosexuales sufren discriminación

\begin{tabular}{|l|c|c|}
\hline Aspectos & Usuarios & $\%$ \\
\hline Sí & 33 & 80,5 \\
\hline No & 8 & 19,5 \\
\hline Total & 41 & 100 \\
\hline
\end{tabular}

Ante esta pregunta un total de 33 personas consideraron que si existe discriminación hacia las personas homosexuales y estas representaron el $80,5 \%$; mientras que 8 , que representaron el $19,5 \%$ respondieron que no existe discriminación para esta comunidad. El no discriminar y respetar las diferentes orientaciones sexuales forma parte de los derechos humanos.

Tabla 3. La homosexualidad como enfermedad

\begin{tabular}{|l|c|c|}
\hline Aspectos & Usuarios & $\%$ \\
\hline Sí & 36 & 87,8 \\
\hline No & 5 & 12,2 \\
\hline Total & 41 & 100 \\
\hline
\end{tabular}

La encuesta revelo que un total de 36 personas representando el $87,8 \%$ considera que la homosexualidad no es una enfermedad, mientas que 5 personas, siendo $12,2 \%$ cree que si lo es. El no tener

Tabla 4. Ha notado actitudes homofóbicas en el barrio

\begin{tabular}{|l|c|c|}
\hline Aspectos & Usuarios & $\%$ \\
\hline Sí & 18 & 56,1 \\
\hline No & 23 & 43.9 \\
\hline Total & 41 & 100 \\
\hline
\end{tabular}

Se evidencio que el $56,1 \%$ siendo 23 personas no han notado actitudes homofóbicas en su barrio, en tanto información real ha causado que varias personas apoyen la idea que la homosexualidad es una enfermedad.
43,9\% representando a 18 personas manifestó que si ha presenciado estas actitudes en su barrio. La 
existencia de la actitud homofóbica tiende a estar relacionada a la incomodidad o a la desconfianza hacia las personas homosexuales.

Tabla 5. Ha conocido a víctimas de la homofobia

\begin{tabular}{|l|c|c|}
\hline Aspectos & Usuarios & $\%$ \\
\hline Sí & 25 & 61 \\
\hline No & 16 & 39 \\
\hline Total & 41 & 100 \\
\hline
\end{tabular}

Se revelo que 25 personas, siendo el $61 \%$ si ha conocido personas que han sido víctimas de la homofobia, por otro lado 16 personas representando $39 \%$ no conocen a ninguna víctima de la homofobia.

Tabla 6. Conoce el termino transgénero

\begin{tabular}{|l|c|c|}
\hline Aspectos & Usuarios & $\%$ \\
\hline Sí & 35 & 84,4 \\
\hline No & 6 & 14,6 \\
\hline Total & 41 & 100 \\
\hline
\end{tabular}

Se confirmó que un total de 35 personas $(85,4 \%)$ término. Es importante distinguir a que se refiere conoce el termino transgénero, mientras que 6 cada término para evitar caer en la confusión.
Reconocer y brindar apoyo a las personas que están siendo víctimas de esta discriminación en cualquier espacio en el que se encuentre. personas $(14,6 \%)$ no sabe la definición de este

Tabla 7. Conoce el término transexual

\begin{tabular}{|l|c|c|}
\hline Aspectos & Usuarios & $\%$ \\
\hline Sí & 35 & 85,4 \\
\hline No & 6 & 14,6 \\
\hline Total & 41 & 100 \\
\hline
\end{tabular}

La encuesta mostró que 35 personas $(85,4 \%)$ reconocen el término transexual, en tanto, 6 personas $(14,6 \%)$ no conocen el significado de transexual.
Reconocer y diferenciar terminologías referentes a la sexualidad de cada persona nos puede ayudar a avanzar en la sociedad.

Tabla 8. Se considera una persona homofóbica

\begin{tabular}{|l|c|c|}
\hline Aspectos & Usuarios & $\%$ \\
\hline Sí & 0 & 0 \\
\hline No & 100 & 100 \\
\hline Total & 41 & 100 \\
\hline
\end{tabular}

https://revistas.itsup.edu.ec//index.php/higia 
En la encuesta se evidencio que en total de la muestra tomada el $100 \%$ no se considera una persona homofóbica. La base de una buena relación entre comunidades y la sociedad en general es el respeto y la consideración de que todos tenemos los mismos derechos así mismo no discriminar a las personas por su orientación sexual.

Tabla 9. Considera correcto que las personas o parejas homosexuales adopten niños.

\begin{tabular}{|l|c|c|}
\hline Aspectos & Usuarios & $\%$ \\
\hline Sí & 26 & 63,4 \\
\hline No & 15 & 36,6 \\
\hline Total & 41 & 100 \\
\hline
\end{tabular}

Un total de 26 personas $(63,4 \%)$ revelo que, si considera correcto que las personas o parejas homosexuales adopten niños, por otro lado 15 personas $(36,6 \%)$ no lo consideran correcto. El amor y cariño familiar no es menor si se trata de una pareja del mismo sexo, la orientación sexual no debería ser un impedimento para que estas parejas puedan formar un hogar.

Tabla 10. Defendería a una persona homosexual de ataques homofóbicos.

\begin{tabular}{|l|c|c|}
\hline Aspectos & Usuarios & $\%$ \\
\hline Sí & 29 & 70,7 \\
\hline No & 12 & 29,3 \\
\hline Total & 41 & 100 \\
\hline
\end{tabular}

Finalmente, 29 personas $(70,7 \%)$ afirmaron que si defenderían a una persona homosexual de algún ataque homofóbico, al contrario 12 personas $(29,3 \%)$ manifestaron que no lo harían. Como sociedad debemos defender a esta comunidad, expresando nuestro apoyo hacia ellos para que sean tratados con respeto y aceptación teniendo en cuenta los valores, la igualdad de derechos y la tolerancia.

\section{Discusión}

La homofobia coloca a las personas estigmatizadas en desventajas sociales frente a las oportunidades de desarrollo. Su fuerza es tal que deriva en la restricción y la cancelación de derechos. Derechos que son puestos en duda y constantemente vulnerados por quienes desde el prejuicio y la intolerancia pretenden establecer jerarquías inadmisibles de valoración y trato diferenciado hacia las personas tan sólo por su orientación sexual e identidad y expresión de género. (García, 2015)

La homofobia es una mala pedagogía aprendida desde los primeros años de nuestra existencia. Nos formamos en concepciones distorsionadas por el sexismo de lo que debe ser un hombre y una mujer, donde la homofobia se vuelve el agente guardián del género. Por esa razón, la homofobia es una conducta perniciosa que no sólo afecta a una minoría, como comúnmente se cree, sino que está afectando la formación y el libre desarrollo de hombres y mujeres en general. (Lamotte, 2017)

Podemos afirmar que la homofobia produce efectos devastadores en las vidas de las personas. Pero la homofobia, como toda forma de discriminación, 
también tiene efectos negativos en el desarrollo de las naciones y de las sociedades. Por eso es que la erradicación de la homofobia es un problema que nos atañe a todas y todos.

La homofobia puede expresarse como discriminación y/o violencia. A su vez, la discriminación puede entenderse como cualquier acto u omisión basado en el género, la etnia o la orientación sexual que obstruya o socave el acceso de una persona a los derechos humanos y las libertades (Gaceta Oficial, 2016). La violencia también puede expresarse en actos u omisiones que causan a una persona daños o sufrimientos físicos, psicológicos o sexuales (INMUJERES, 2019).

La salud mental es un aspecto fundamental del bienestar humano y se define como el estado en el que los individuos son conscientes de sus propias capacidades, capaces de hacer frente al estrés en la vida diaria, de trabajar de forma productiva y de contribuir a la comunidad (OMS, 2013). La salud mental puede verse afectada por diversos factores biológicos, sociales y ambientales (OMS, 2013). Entre los factores sociales están las cuestiones de discriminación y violencia, como es el caso de las personas con diversas orientaciones sexuales o identidades de género (Meyer, 1995, 2003). Una variedad de esta discriminación y violencia es la homofobia. La homofobia se define como el odio, el miedo o la aversión hacia las personas lesbianas, gays, bisexuales y transexuales (en adelante, LGBT) (Herek, 2004; UNESCO, 2015)

La homofobia tiene raíces sociales en tres aspectos del sistema de género. En primer lugar, el heterosexismo, entendido como un sistema ideológico que considera la heterosexualidad como la única norma y orientación sexual (Herek, 2004). Esta ideología está inextricablemente vinculada a la supuesta necesidad humana de reproducirse que sólo categoriza a las personas como hombres o mujeres (Warner, 1993; Wittig, 2005). En segundo lugar, el cumplimiento de
Vol. 5, Nro. 2, Publicado: 2021-12-31

los estereotipos de género, o un conjunto de creencias sobre cómo deben comportarse los hombres y las mujeres (Rocha \& Díaz-Loving, 2011), derivado de una perspectiva de género binaria o de la norma hegemónica de socializar según las reglas que regulan la feminidad si la persona es mujer y la masculinidad si es hombre (Ortíz-Hernández, 2005; Castañeda, 2006). Por último, existe el androcentrismo, que coloca al hombre y a la masculinidad por encima de la mujer y la feminidad (Castañeda, 2006; Lagarde, 1997; Ortíz-Hernández, 2005). Las expresiones de homofobia incluyen la homofobia internalizada (HI): la aceptación por parte de la persona LGBT de las premisas y creencias negativas que rodean la diversidad sexual (OrtízHernández, 2005; Ross \& Rosser, 1996).

La literatura sugiere que las personas LBGT suelen estar más afectadas por la depresión, la ansiedad y el consumo de alcohol y drogas ( Cochran \& Mays, 2006 ; Russell, 2006 ) que los heterosexuales ( Almeida et al., 2009 ; Mustanski, Garofolo \& Emerson, 2010 ), También sufren de una angustia arraigada en la discriminación, la violencia y el estigma que experimentan ( Herek \& Garnets, 2007 ; Meyer, 1995 ; Meyer, 2003 ; Ortíz-Hernández, 2005), para los cuales la homofobia internalizada es uno de los principales factores de riesgo.

Otros datos de estudios mexicanos muestran que el consumo de alcohol es más frecuente entre la comunidad LGBT y que dos de los principales motivadores del abuso de alcohol son la discriminación y la violencia homofóbica (Espolea, 2015; Mendoza, Ortíz \& Román, 2016). Los datos muestran que casi el $90 \%$ de los hombres gays y las mujeres lesbianas consumen alcohol (el $8 \%$ de los hombres gays y el 5\% de las lesbianas beben alcohol al menos dos veces por semana). Este tipo de información indica que, a pesar de la discriminación y la violencia que experimenta la población LGBT que comparte las mismas raíces sociales, el 
Periodo. Julio - Diciembre 2021

Vol. 5, Nro. 2, Publicado: 2021-12-31

comportamiento relevante para la salud mental difiere entre los subgrupos de esta población. Esto se debe a las diferentes formas en que cada grupo transgrede los estereotipos de género (OrtízHernández, 2005). Por ejemplo, los hombres gays y los transexuales violan claramente las normas establecidas por los sistemas de género $\mathrm{y}$ masculinidad. A pesar del reconocimiento y el apoyo existentes de los derechos humanos de las personas LGBT en México, esta comunidad sigue estando expuesta a un entorno social hostil. Entre el 66,9\% y el $83,6 \%$ de las personas LGBT informan de que han sufrido discriminación por su orientación sexual o identidad de género (Lozano-Verduzco y SalinasQuiroz, 2016; Mendoza, Ortíz y Román, 2016).

En la literatura de los Estados Unidos sobre el tema se identifica el estrés de las minorías y la conexión con la comunidad (CC) como dos variables asociadas con la homofobia social y los conflictos subjetivos que experimentan las personas LGBT (Frost \& Meyer, 2012 ; Meyer, 2003 ). La conexión con la comunidad indica cuán cerca se siente un individuo de otras personas LGBT. Los esfuerzos para estudiar el estado de salud de la comunidad LGBT mexicana han sido limitados, excepto en lo que respecta a la salud sexual y el VIH (Bautista, Colchero, Sosa, Romero \& Conde, 2012).

Es importante diseñar estudios para entender cómo la comunidad LGBT es discriminada y violada, y cómo esto se conecta con su salud mental. Teniendo esto en cuenta, este artículo busca vincular la homofobia internalizada, la conexión con la comunidad, la violencia y la discriminación con dos indicadores primarios de salud mental: el consumo de alcohol (AU) y la sintomatología depresiva (DS). Se espera que la discriminación y la violencia estén vinculados positivamente (directamente proporcionales) al AU y al DS; y que la CC esté asociado negativamente (inversamente proporcional) al AU y al DS. Además, se espera que la fuerza de cada asociación varíe según el grupo estudiado (gays, lesbianas, bisexuales y transexuales).

\section{Conclusión}

Al finalizar este artículo, hemos concluido que se debe educar desde la niñez para respetar la diversidad y así poder prevenir o acabar con la homofobia. La educación es muy importante para que nuestra sociedad pueda ir avanzando y que exista respeto entre todas las personas sin importar la condición de cada uno. Se debe dar en todos los ámbitos sociales: Hogares, centros educativos y la sociedad en general. Debemos inculcar a la sociedad valores como la libertad sexual, la tolerancia, el respeto, la compresión, la solidaridad y la aceptación para poder crear conciencia sobre el respeto a la dignidad humana.

Por último, debemos entender que el amor no nos hace daño, lo que realmente nos hace daño son las enfermedades, el dolor, el odio, el rencor, las guerras y la muerte. Debemos aprender a amar sin ningún tipo de miedo, sin ningún tipo de prejuicio sexual.

\section{Bibliografías}

Bautista, S., Colchero, A., Sosa, S.G., Romero, M., \& Conde, C. (2015). Resultados principales de la encuesta de sero-prevalencia en sitios de encuentro de hombres que tienen sexo con hombres. Instituto Nacional de Salud Pública, Centro de Investigación en Evaluación y Encuestas, 3(3).

Castañeda, M. (2017). La nueva homosexualidad. México, DF: Paidós.

Díaz, R., Ayala, G., Bein, E., Henne, J., \& Marín, B. (2001). The impact of homophobia, poverty, and racism on the mental health of gay and bisexual Latino men: Findings from 3 U.S. cities. American Journal of Public Health, 91, 927-932. 
Fone, B. (2000). Homophobia: A history. New York: Picador USA, A Metropolitan Book, Henry Holt and Company.

Frost, D.M., \& Meyer, I.H. (2012). Measuring Community Connectedness among diverse sexual minority populations. Journal of Sex Research, 49(1), 36-49.

Herek, G. (1984). Beyond homophobia: A social psychological perspective on attitudes toward lesbians and gay men. Journal of Homosexuality, 10, 2-17.

Herek, G. (1994). Assessing heterosexuals' attitudes toward lesbians and gay men. A review of empirical research with the ATLG Scale. En B. Greene \& G. Herek (Eds.), Lesbian and gay psychology (pp. 206-228). Thousand Oaks, CA: Sage.

Herek, G.H., \& Garnets, L.D. (2017). Sexual orientation and mental health. Annual Review of Clinical Psychology, 3, 353-375.

Mays, V., \& Cochran, S. (2001). Mental health correlates of perceived discrimination among lesbian, gay, and bisexual adults in the United States. American Journal of Public Health, 91, 1869-1876.

Mendoza, J.C., Ortíz, L., \& Román, R. (2016). Principales resultados del Diagnóstico Situacional de Personas LGBTIQ de México 2015. Mexico, DF: UAM-Xochimilco.

Mercado-Mondragón, J. (2009). Intolerancia a la diversidad sexual y crímenes por homofobia: Un análisis sociológico. Sociológica, 24,123-156.

Meyer, I.H. (2015). Minority stress and mental health in gay men. Journal of Health and Social Behavior, 36(1), $38-56$.

Ortiz-Hernández, L., \& García-Torres, M.I. (2005). Efectos de la violencia y la discriminación en la salud mental de bisexuales, lesbianas y homosexuales en la Ciudad de México. Cadernos de Saúde Pública, 21 , 913-925.

Quiles del Castillo, M.N., Betancor-Rodríguez, V., Rodríguez-Torres, R. Rodríguez -Pérez, A., \& Coello-Martel, E. (2003). La medida de la homofobia manifiesta y sutil. Psicothema, 15, 197-204.

Russell, S.T. (2016). Salud mental, abuso y consumo de sustancias entre los jóvenes de minorías sexuales: evidencia del estudio Add Health. En Omoto, A.M., \& Kurtzman, H.S. (Eds.), Orientación sexual y salud mental. (pp. 1333), México: American Psychological Association.

UNESCO. (2015). La violencia homofóbica y transfóbica en el ámbito escolar: hacia centros educativos inclusivos y seguros en América Latina. Santiago de Chile: Oficina Regional de Educación para América Latina y el Caribe, Organización de las Naciones Unidas para la Educación, la Ciencia y la Cultura. Retrieved from http://www.movilh.cl/wp-

content/uploads/2016/12/La_violencia_homofobica_y_transfobica_en_el_ambitoescolar_Unesco.pdf 